\title{
Antitumor effects of telomerase-specific replication-selective oncolytic viruses for adenoid cystic carcinoma cell lines
}

\author{
DAISUKE SATO $^{1}$, YUJI KURIHARA ${ }^{1 *}$, SEIJI KONDO $^{1 *}$, TATSUO SHIROTA $^{1}$, \\ YASUO URATA $^{2}$, TOSHIYOSHI FUJIWARA ${ }^{3,4}$ and SATORU SHINTANI ${ }^{1}$ \\ ${ }^{1}$ Department of Oral and Maxillofacial Surgery, School of Dentistry, Showa University, Ota-ku, Tokyo 145-8515; \\ ${ }^{2}$ Oncolys BioPharma, Inc., Minato-ku, Tokyo 106-0032; ${ }^{3}$ Division of Surgical Oncology, Department of Surgery, \\ Okayama University, Graduate School of Medicine and Dentistry, Kita-ku, Okayama 700-8558; ${ }^{4}$ Center for Gene \\ and Cell Therapy, Okayama University Hospital, Kita-ku, Okayama 700-8558, Japan
}

Received July 1, 2013; Accepted August 14, 2013

DOI: $10.3892 /$ or.2013.2738

\begin{abstract}
We evaluated the antitumor effect of a telomerasespecific replication-selective adenovirus (Telomelysin, OBP-301) for adenoid cystic carcinoma (ACC) in vitro and in vivo. Adenovirus E1 gene expression was controlled by human telomerase reverse transcription (hTERT). Infection of ACC cells by OBP-301 induced high E1A mRNA expression and subsequent oncolytic cell death in a dose-dependent manner. Using OBP-401 (TelomeScan), a genetically engineered adenovirus that carries the GFP gene under the control of the cytomegalovirus (CMV) promoter at the deleted E3 region of OBP-301, ACC cells expressed bright GFP fluorescence as early as $12 \mathrm{~h}$ after OBP-401 infection. The fluorescence intensity gradually increased in a timedependent manner, followed by rapid cell death due to the cytopathic effect of OBP-401, as evidenced by the floating, highly light-refractive cells using phase-contrast microscopy. Effects of intratumorally injected OBP-401 against established Acc2 xenograft tumors were seen in BALB/c $n u / n u$ mice. The levels of GFP expression following ex vivo infection of OBP-401 may be of value as a positive predictive marker for the outcome of telomerase-specific virotherapy. Our data clearly indicated that telomerase-specific oncolytic adenoviruses have significant therapeutic potential against human ACC in vitro and in vivo. These results suggest that treatment with OBP-301 and OBP-401 may improve the quality of life of oral cancer patients.
\end{abstract}

Correspondence to: Dr Yuji Kurihara, Department of Oral and Maxillofacial Surgery, School of Dentistry, Showa University, 2-1-1 Kitasenzoku, Ota-ku, Tokyo 145-8515, Japan

E-mail: ykurihara@dent.showa-u.ac.jp

*Contributed equally

Key words: antitumor, telomerase, telomerase-specific replicationselective oncolytic viruses, adenoid cystic carcinoma

\section{Introduction}

ACC is a malignant neoplasm that originates in both the minor and major salivary glands, the naso-pharyngeal secretory gland and the lacrimal gland with some other rare primary sites, and is characterized by slow growth, diffuse invasion, a high tendency towards mass formation and local recurrence, and the potential to produce distant metastases, mainly to the lungs and bones $(1,2)$. Late distant metastases are, together with local recurrences, responsible for the rather low long-term survival rate (3). ACC of the salivary gland is an uncommon malignancy, comprising only $1 \%$ of head and neck tumors, $10 \%$ of salivary malignancies and $22 \%$ of all salivary gland malignancies $(4,5)$. Many types of combination chemotherapy and molecular target therapy have been investigated; however, only limited responses have been shown (5).

The telomerase-specific replication-competent adenovirus (Telomelysin, OBP-301), in which the human telomerase reverse transcriptase (hTERT) promoter element drives expression of the E1A and E1B genes linked with an internal ribosomal entry site (IRES), induces selective E1 expression (6-9). Moreover, $>85 \%$ of human cancers display telomerase activity (10), and both telomerase activity and hTERT expression are increased in human salivary gland carcinomas. Intratumoral injection of OBP-301 in combination with a replicationdeficient adenovirus expressing the green fluorescent protein (GFP) gene (OBP-401, TelomeScan) causes viral spreading into the regional lymphatic area with subsequent selective replication in metastatic lymph nodes in BALB/c nu/nu mice (11). Furthermore, we showed that OBP-301 induces a selective antitumor effect in OSCC cells in vitro and in an in vivo orthotopic graft model (12). However, the antitumor effects of these telomerase-specific replication-selective oncolytic viruses are not yet fully understood in the framework of ACC. In the present study, we investigated the in vitro and in vivo inhibitive efficacy of OBP-301 and OBP-401 against ACC.

\section{Materials and methods}

Cell lines and cell culture. Human salivary gland adenoid cystic carcinoma cell lines (Acc2 and AccM) were kindly 
provided by Dr W.L. Qiu. The Acc2 cell line was derived from a 28-year-old female patient, and AccM was established from Acc2 as a highly metastatic subclone (13,14). Acc2, AccM and the OSCC cell line SAS were maintained in vitro as monolayers at $37^{\circ} \mathrm{C}$ with $5 \% \mathrm{CO}_{2}$ in Dulbecco's modified Eagle's medium (DMEM) supplemented with $10 \%$ fetal bovine serum, 100 units $/ \mathrm{ml}$ penicillin and $100 \mathrm{mg} / \mathrm{ml}$ streptomycin.

Adenoviruses. The telomerase-specific replication-selective oncolytic virus vector OBP-301 (Telomelysin) was constructed and previously described; the hTERT promoter element drives expression of the $E 1 A$ and $E 1 B$ genes linked with an internal ribosome entry site (IRES). A GFP gene driven by the cytomegalovirus (CMV) promoter was inserted into OBP-301, so that the virus would express GFP during efficient replication in infected cells $(7,9,15)$.

OBP-401 (TelomeScan) is a telomerase-specific replication-selective oncolytic virus variant, in which a GFP gene under control of the cytomegalovirus promoter is inserted into the deleted E3 region of OBP-301 for monitoring viral replication (11). The viruses were purified by $\mathrm{CsCl}_{2}$ linear gradient ultracentrifugation. The viral titers were determined by plaque-forming assay using 293 cells and then stored at $-80^{\circ} \mathrm{C}$.

Western blot analysis. Protein was extracted from collected cells with Triton X-100 lysis buffer (50 mM Tris $\mathrm{pH} 8.0$, $300 \mathrm{mM} \mathrm{NaCl}, 0.5 \%$ Triton X-100, $5 \mathrm{mM}$ EDTA, $1 \mathrm{mM}$ sodium $o$-vanadate) supplemented with Complete Mini (Roche Diagnostics, Basel, Switzerland) protease inhibitor tablets. Cells were lysed after $48 \mathrm{~h}$ of cell seeding, and protein concentrations were subsequently measured using the Bio-Rad Quick Start protein assay (Bio-Rad Laboratories, Hercules, CA, USA). Western blot analysis of fraction markers was carried out according to the method previously described (16). The proteins in the SDS sample buffer (25 $\mu \mathrm{g}$ protein) were heated, separated on 4-15\% Mini Protean TGX precast gels (Bio-Rad Laboratories) and transferred to a polyvinylidene difluoride (PVDF) membrane (Life Technologies, Billerica, MA, USA). After the membrane was blocked with $0.3 \%$ skim milk, the primary antibodies against hTERT (Merck Millipore, Billerica, MA, USA), CAR, GAPDH (Santa Cruz Biotechnology, CA, USA) and subsequently, peroxidase-linked secondary antibodies (GE Healthcare, Buckinghamshire, UK) were used. The blots were visualized using the ECL Western blotting detection system (GE Healthcare).

Cell viability assay. Acc2, AccM and SAS cells were seeded on 96-well plates at a density of $2.5 \times 10^{3}$ cells/well $18-24 \mathrm{~h}$ before viral infection. Cells were infected with OBP-301 at multiplicity of infection (MOI) of $1,10,50$ and 100 plaqueforming units (PFU)/cell. Cell viability was determined by an XTT assay at 24, 48, 72 and $120 \mathrm{~h}$ using the Cell proliferation kit II (Roche Diagnostics) according to the protocol recommended by the manufacturer; the particular XTT was sodium 3'-[1-(phenylamino-carbonyl)-3,4-tetrazolium]-bis (4-methoxy-6-nitro)benzene-sulfonic acid hydrate.

Quantitative real-time RT PCR analysis. Acc2 and AccM cells, seeded on $100-\mathrm{mm}$ dishes at a density of $4 \times 10^{5}$ cells 18-24 h before viral infection, were infected with OBP-301 at
$50 \mathrm{PFU} /$ cells. Following the removal of the virus, cells were further incubated and harvested 2, 24, 48 and $72 \mathrm{~h}$ later, and total RNA was extracted using TRIzol (Life Technologies). cDNA was generated from $500 \mathrm{ng}$ of total RNA using the iScript cDNA synthesis kit (Bio-Rad Laboratories). Quantitative real-time PCR for the E1A gene was performed using the iCycler iQ Real-time PCR detection system (Bio-Rad Laboratories) and the iQ SYBR-Green Supermix (Bio-Rad Laboratories), according to the manufacturer's instructions. The primer sequences of E1A used in the present study were as follows: forward, 5'-GCT GAT CGA AGA GGT ACT GC-3' and reverse, 5'-ACC GCC AAC ATT ACA GAG TC-3'. The cDNA amplification program for E1A genes consisted of denaturation at $95^{\circ} \mathrm{C}$ for $2 \mathrm{~min}$, followed by 45 cycles at $95^{\circ} \mathrm{C}$ for $5 \mathrm{sec}$ and annealing and extension at $60^{\circ} \mathrm{C}$ for $20 \mathrm{sec}(17)$.

Fluorescence microscopy. Acc 2 and AccM cells were infected with OBP-401 at the indicated MOI values in a 6-well flat-bottomed culture plate and further incubated for the indicated time periods. The expression of GFP fluorescence was measured and photographed at the magnification of $\mathrm{x} 20$ using a fluorescence microplate reader (BioStation CT; Nikon, Corp., Tokyo, Japan).

Animal experiments. Acc 2 and AccM cells ( $3 \times 10^{6}$ cells/mouse) suspended in Matrigel were injected into the submandibular glands of female 6-week-old BALB/c $n u / n u$ nude mice to generate a subcutaneous tumor model. When tumors reached 3-5 mm in diameter, OBP-301 or OBP-401 at a dose of $1 \times 10^{8}$ plaque-forming units (PFU)/tumor or PBS was injected into the tumor (12).

The perpendicular diameter of the tumors was measured every 3 days, and the tumor volume was calculated using the following formula: Tumor volume $\left(\mathrm{mm}^{3}\right)=a \times b^{2} \times 0.5$, where $\mathrm{a}$ is the longest diameter, $\mathrm{b}$ is the shortest diameter and 0.5 is a constant to calculate the volume of an ellipsoid. The body weights of mice were monitored and recorded. The experimental protocol was approved by the Ethics Review Committee for Animal Experimentation at Showa University.

\section{Results}

In vitro cytopathic effect of OBP-301 on ACC cell lines and the SCC cell line, CAR and hTERT protein expression and E1A RNA expression. Western blot analysis revealed the protein expression levels of CAR and hTERT in ACC and SCC cell lines (Fig. 1A). The in vitro cytopathic effect of OBP-301 on ACC cell lines and the human SCC cell line by XTT assay is shown (Fig. 1B). ACC and SCC cell lines were infected with OBP-301 at various MOIs for 5 days. OBP-301 infection induced cell death in a time-dependent manner. Acc 2 showed the highest expression levels of CAR and AccM showed the highest expression of hTERT. E1A expression in OBP-301infected Acc 2 and AccM cell lines multiplied after $48 \mathrm{~h}$ when OBP-301 at a MOI of 50 was used (Fig. 1C).

Visualization of OBP-401 in ACC cell lines in vitro. Tumorspecific replication-competent adenovirus OBP-401 contains the GFP gene under control of the CMV promoter at the deleted E3 region of the telomerase-specific replication-selective 
A

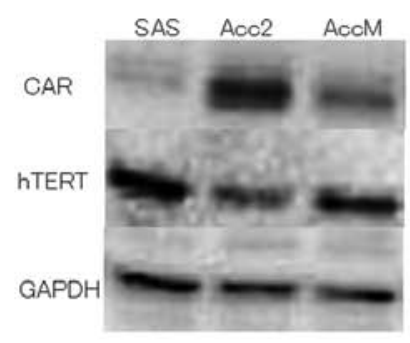

C

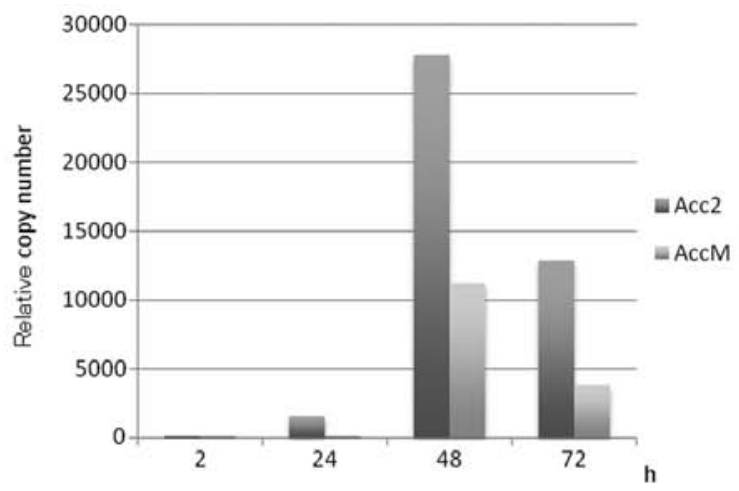

B

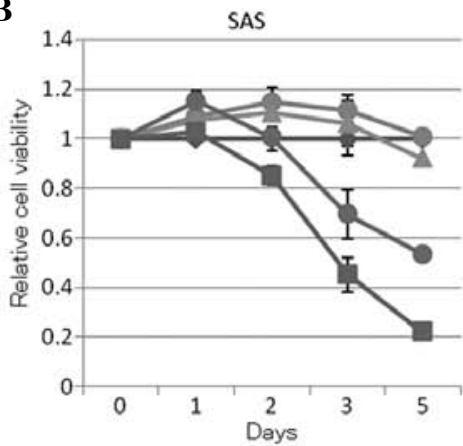

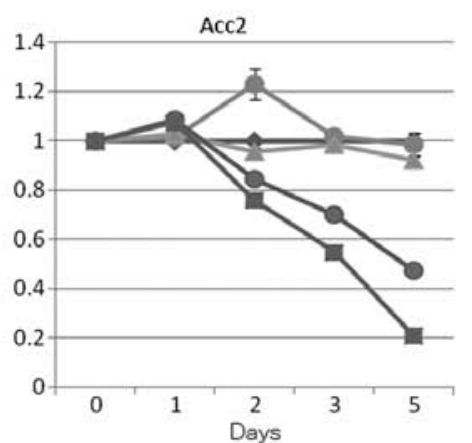

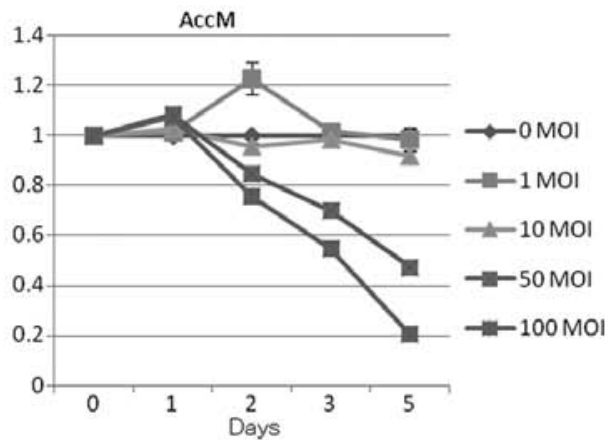

Figure 1. Expression of CAR and hTERT, antitumor activity of OBP-301, and E1A expression in OBP-301 infected ACC cells. (A) Western blot analysis for CAR and hTERT. (B) ACC cell lines, Acc2 and AccM and the OSCC cell line SAS were infected with OBP-301 at the indicated MOI values, and surviving cells were quantitated over 5 days by the XTT assay. The cell viability of mock-treated cells on day 0 was considered to be 1.0 as a basis for the calculation of relative cell viability. Points, mean of triplicate experiments; bars, SD. (C) E1A expression OBP-301 at a MOI of 50 infected Acc2 and AccM cells. Relative replication rate normalized by dividing the value of cells obtained $2 \mathrm{~h}$ after viral infection. By comparison, Acc2 values were higher than AccM at all time-points.
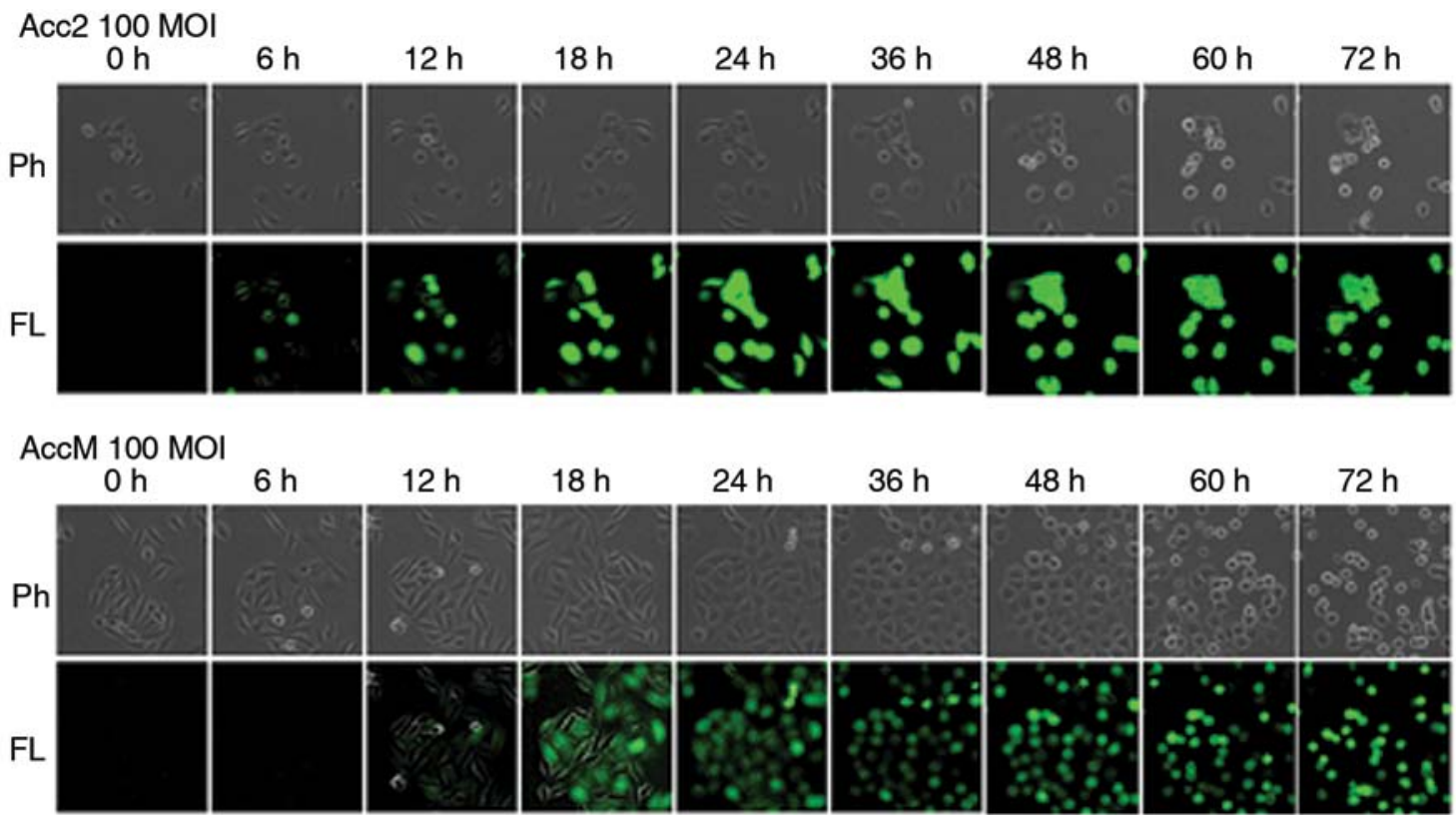

Figure 2. Selective visualization of ACC cell lines in vitro after infection with OBP-401. Cell morphology was evaluated at the indicated time-points by phase-contrast microscopy (upper panel) and GFP expression under fluorescence microscopy (lower panel). Cells were infected with OBP-401 at a MOI of 100 .

type 5 adenovirus, OBP-301. To determine whether OBP-401 replication is associated with selective GFP expression, ACC cell lines were analyzed by fluorescence microscopy after OBP-401 infection. Acc 2 and AccM cells expressed bright GFP fluorescence as early as 6 and 12 h, respectively, after
OBP-401 infection at a MOI of 100. The fluorescence intensity gradually increased in a dose-dependent manner (Fig. 2).

In vivo studies of antitumor effects of intratumoral injection of OBP-401 in a xenograft mouse model. We investigated 


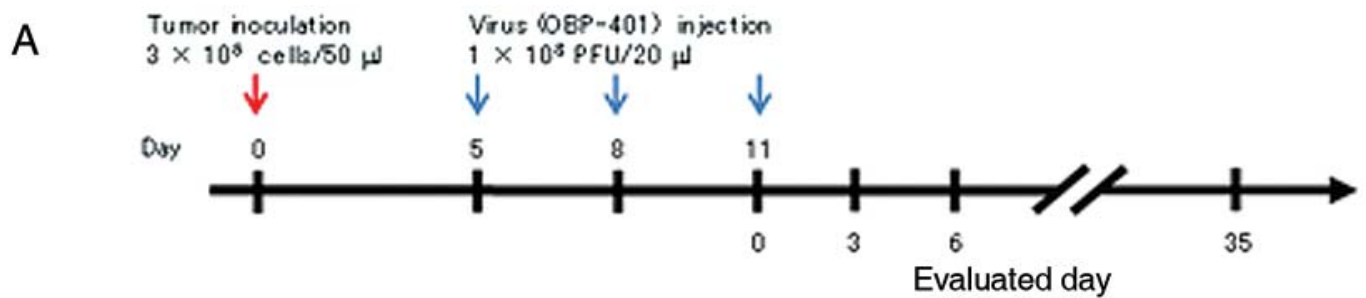

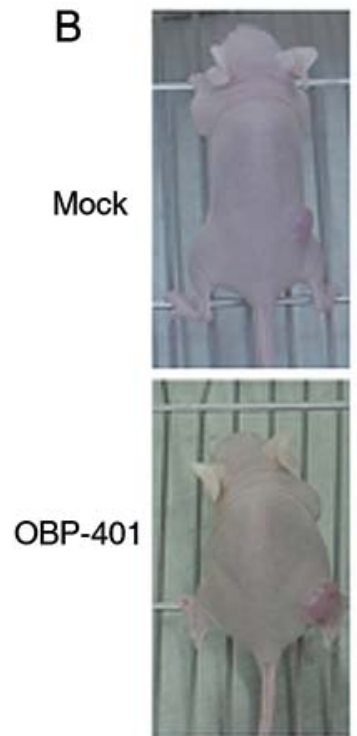

Day 0

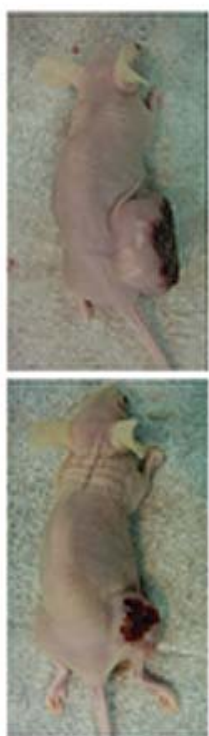

Day 35
C
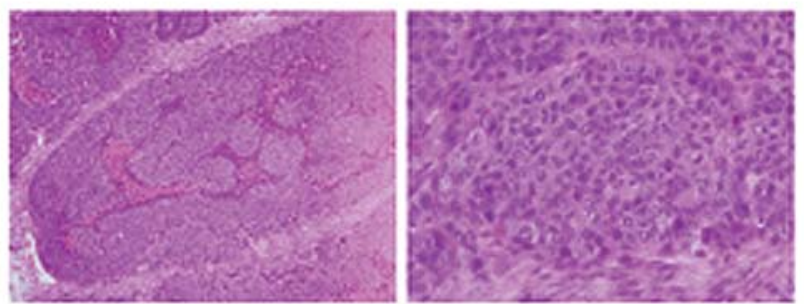

D

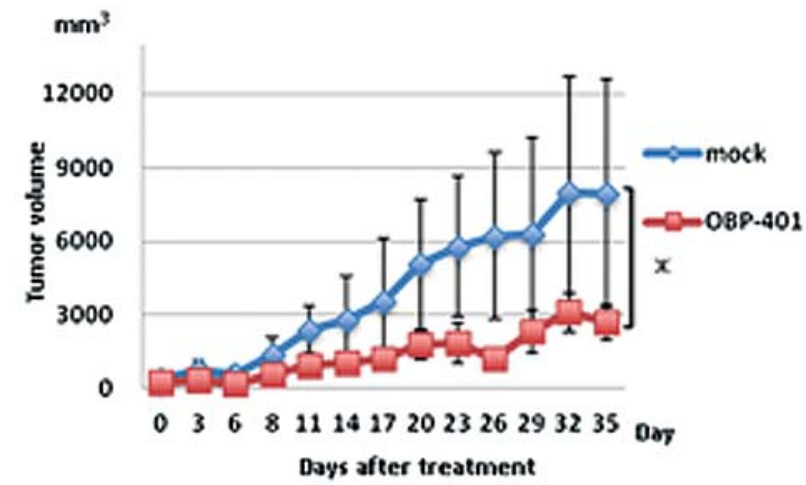

Figure 3. Antitumor effect of OBP-401 on Acc2 cells in a xenograft model. (A) Protocol of a xenograft model experiment. BALB/c $n u / n u$ mice were subcutaneously inoculated with $3 \times 10^{6}$ Acc2 cells. Tumor-bearing mice received three intratumoral injections of $1 \times 10^{8}$ PFU of viruses every 3 days starting day 5 after tumor cell inoculation. Six mice were used in each group. (B) Macroscopic appearance of ACC2 xenograft mouse mock- (top) or OBP-401-inoculated (bottom) after 0 (left) and 35 days (right). (C) Paraffin-embedded sections of Acc2 subcutaneous tumors were stained with H\&E. Magnification: Left panel, x40; right panel, $\mathrm{x}$ 400. (D) The tumor volume was monitored and plotted. Point, mean; bars, SD. Statistical significance was defined as P<0.05 (Student's t-test).
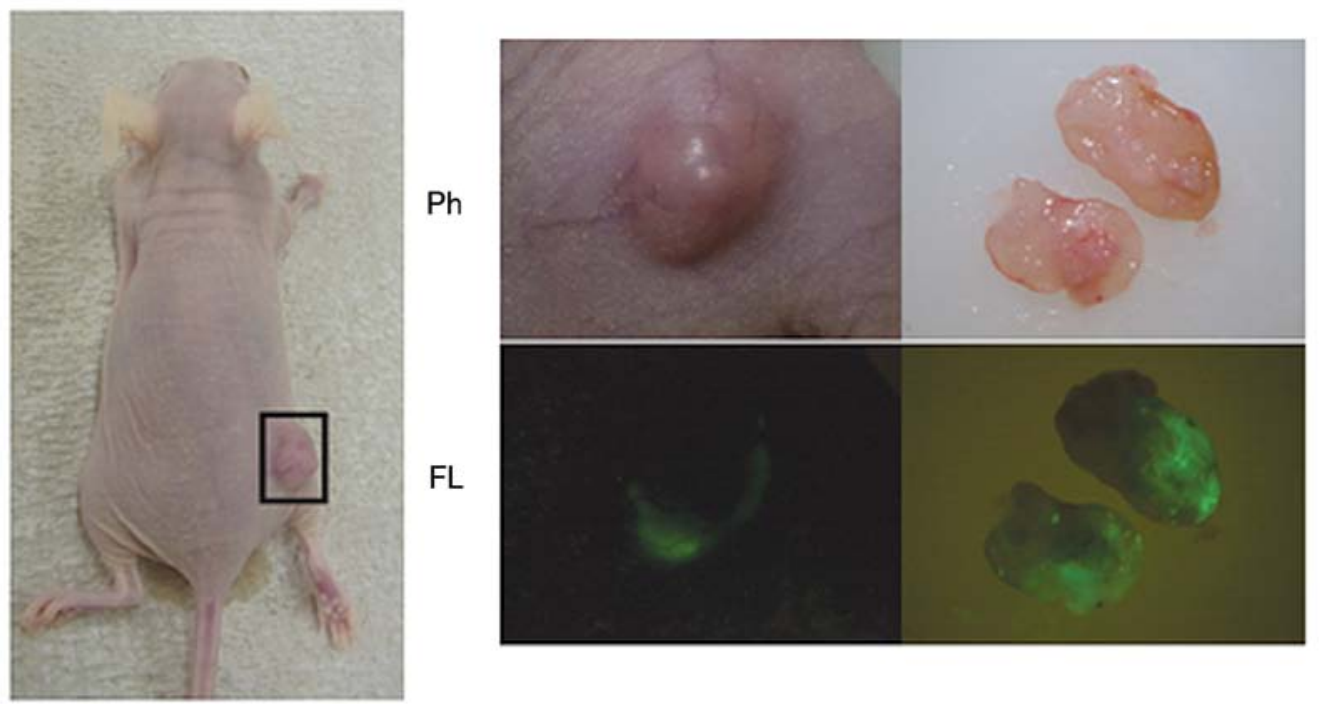

Figure 4. Selective visualization of Acc2 cells in vivo by OBP-401. Mice received intratumoral injections of OBP-401 (1x10 $\mathrm{PFU}$ ) three times after tumor inoculation and were assessed 14 days later under imaging by a charge-coupled device. Macroscopic appearance (top) of subcutaneous tumor and fluorescence image (bottom).

the antitumor activity of OBP-401 against Acc2 cells in vivo. We used an animal model for ACC in which Acc2 cells were implanted into the flank of BALB/c nu/nu mice. Mice bearing palpable Acc2 tumors with diameters of 5-10 mm received three courses of intratumoral injections of $10^{8} \mathrm{PFU}$ of OBP-401 or PBS (mock treatment) every 3 days after the initial tumor 
inoculation (Fig. 3A). Representative images from each group showed that tumors treated with OBP-401 three times with starting day 11 after tumor inoculation were smaller than those of mock-treated mice 35 days after the third viral infection (Fig. 3B). Histopathological examination of the excised primary tumors showed a tumor composed of implanted Acc2 cells with a solid architecture (Fig. 3C). Representative images from each group showed that tumors treated with OBP-401 with starting day 5 after tumor inoculation were consistently smaller than those of mock-treated mice 35 days after the last viral injection $(\mathrm{P}<0.05)$ (Fig. 3D).

Selective visualization of infected viruses in subcutaneous tumors in vivo. We visualized OBP-401-infected Acc2 cells intratumorally in BALB/c nu/nu mice. Tumor-bearing mice, inoculated subcutaneously with $3 \times 10^{6}$ Acc2 cells, were observed 14 days after treatment with OBP-401 for the third time (Fig. 4, left panel). GFP signals were detected through the skin (Fig. 4, middle panel), showing that adenoviruses had spread into the surgically removed tissue (Fig. 4, right panel).

\section{Discussion}

Telomerase is expressed in a majority of human cancers, and its activation plays a critical role in tumorigenesis by sustaining cell immortality (18). Telomerase activity is detected in about $85 \%$ of human cancers (19), whereas telomerase is absent in most normal somatic tissues (20). The adenovirus E1B gene is expressed early in viral infection and its gene product inhibits E1A-induced p53-dependent apoptosis, which in turn promotes the cytoplasmic accumulation of late viral mRNA, leading to a shutdown of host cell protein synthesis. In most vectors that replicate under the transcriptional control of the $E 1 A$ gene, including hTERT-specific oncolytic adenoviruses, the $E 1 B$ gene is driven by the endogenous adenovirus E1B promoter (15). In OBP-301, the tumor-specific hTERT promoter regulates both the $E 1 A$ and $E 1 B$ genes. OBP-301 is expected to control its replication more stringently, thereby providing better therapeutic effects in tumor cells as well as attenuated toxicity in normal tissue (6).

The sensitivity varied greatly between cells despite human telomerase reverse transcriptase and CAR protein expression. The hTERT promoter is reported to be inactive in normal cells. It may provide transcriptional activity in some cell types with telomerase activity, such as stem or germline cells $(21,22)$. In fact, protein expression of CAR was higher in SAS than in Acc2 or AccM, and AccM protein expression of hTERT was higher than that of Acc2 (Fig. 1A). Expression levels of CAR and hTERT showed no significant difference between AccM and SAS cells; nevertheless, the anticancer effect of OBP-301 on the SAS cell line was better than that on AccM cells by the XTT assay. Thus, neither hTERT nor CAR protein expression levels appear to be useful for predicting the outcome of OBP-301 treatment. We reported that OBP-301 showed an antitumor effect on Acc2 cells in a dose-dependent manner (Fig. 1B). In vitro, OBP-301 has been shown to kill various human cancer cell lines, as determined by cell proliferation assay $(6,9,17,23,24)$; moreover, a previous study showed no significant toxicity of OBP-301 to normal human cells $(11,25,26)$. E1A is the first adenoviral gene to be expressed; therefore, quantitative real-time RT-PCR analysis of E1A reveals intracellular amplification of OBP-301 (Fig. 1C). As E1A expression correlates directly with overall virus efficacy, the ability of the host cell to permit expression would profoundly influence all subsequent parts of the life cycle. However, previous studies found that E1A expression does not correspond with CAR and hTERT expression; hence, the levels of expression vary widely and do not correlate with OBP-301 sensitivity $(12,27)$. Furthermore, a recent report showed that OBP-702, in which a human wild-type p53 gene expression cassette was inserted into the E3 region of OBP-301 suppressed the viability of cancer cells more efficiently than OBP-301, inducing dual apoptotic and autophagic cell death $(28,29)$. This novel apoptotic mechanism suggests that the p53-expressing OBP-702 also has a possibility of antitumor efficacy for adenoid cystic carcinoma cells.

In the present study, we inoculated tumor cells subcutaneously into BALB/c nu/nu mice and confirmed the formation of tumors with diameters of 5-9 mm after 5 days. We showed that OBP-401 has antitumor activity against ACC tumors in vivo (Fig. 3B and $\mathrm{D}$ ). When tumors of OBP-401 treated mice $\left(2,694 \mathrm{~mm}^{3}\right)$ were compared with those of non-treated mice $\left(7,945 \mathrm{~mm}^{3} ; \mathrm{P}<0.05\right)$, the results indicated that their size clearly decreased after 35 days. Acc 2 cell lines examined in our preliminary experiments were susceptible to telomerasespecific replication-selective oncolytic virus infection in vitro and in vivo, suggesting that the proportion of potentially treatable cancer is high. Recently, OBP-301 has been developed as an oncolytic viral agent for the treatment of human cancer and is currently being evaluated in a Phase-I clinical trial (30). On the basis of these results, and with future clinical applications in mind, we established a therapeutic strategy for the use of telomerase-specific oncolytic adenoviruses to treat patients with adenoid cystic carcinoma. This strategy involves assessment of the expression levels of CAR and hTERT in human ACC cells, which would then allow easy selection of the most effective protocol for the treatment of patients with oncolytic adenoviruses.

We demonstrated GFP expression following OBP-401 in vitro and in vivo (Figs. 2 and 4). Administration of OBP-401 can provide an additional advantage in cancer therapy. OBP-401, similar to OBP-301, is an oncolytic virus and selectively kills human tumor cells by viral replication. Tumor cells infected with OBP-401 express GFP fluorescence and then lose viability, allowing the timing of detection. We speculate that OBP-401 would spread into the regional lymph nodes after intratumoral injection $(11,12)$, express GFP signals in tumor cells by virus replication and finally kill tumor cells even if the surgeon failed to remove all nodes containing micrometastasis. Thus, the oncolytic activity of OBP-401 may function as a backup safety antitumor program. Further prospective clinical studies are required to confirm the direct correlation between GFP expression in biopsy samples following ex vivo OBP-401 infection and the clinical response to OBP-401 in patients with ACC.

In conclusion, our data clearly indicate that telomerasespecific replication-selective oncolytic adenoviruses can significantly inhibit ACC cell growth in vitro and in vivo. Moreover, these viruses can be used in an ex vivo diagnostic assay to predict the therapeutic potential of viruses in ACC 
patients (31). This novel technology will affect and contribute to the minimum operative procedure for ACC cancer patients.

\section{Acknowledgements}

The authors wish to thank Drs Yoshiki Mukudai and Sunao Shiogama for helpful suggestions and Ms. Miho Yoshihara for her secretarial assistance. The present study was supported by grants-in-aid for Scientific Research from the Japan Society for the Promotion of Science (to S.K. and Y.M.) and the High-Technology Research Center Project from the Ministry of Education, Culture, Sports, Science and Technology (to S.S.).

\section{References}

1. Umeda M, Komatsubara H, Nishimura N, Oku N, Shibuya Y, Yokoo S and Komori T: Establishment and characterization of a human adenoid cystic carcinoma line of the salivary gland which is serially transplantable and spontaneously metastasizes to the lung in nude mice. Oral Oncol 38: 30-34, 2002.

2. Alleyne CH, Bakay RA, Costigan D, Thomas B and Joseph GJ: Intracranial adenoid cystic carcinoma: case report and review of the literature. Surg Neurol 45: 265-271, 1996.

3. van der wal JE, Becking AG, Snow GB and van der Wall I: Distant metastases of adenoid cystic carcinoma of the salivary glands and the value of diagnostic examinations during followup. Head Neck 24: 779-783, 2002.

4. Mithani SK, Shao C, Tan M, Smith IM, Califano JA, El-Naggar AK and Ha PK: Mitochondrial mutations in adenoid cystic carcinoma of the salivary glands. PLoS One 4: e8493, 2009.

5. Dodd RL and Slevin NJ: Salivary gland adenoid cystic carcinoma: a review of chemotherapy and molecular therapies. Oral Oncol 42: 759-769, 2006

6. Kawashima T, Kagawa S, Kobayashi N, et al: Telomerase-specific replication-selective virotherapy for human cancer. Clin Cancer Res 10: 285-292, 2004.

7. Taki M, Kagawa S, Nishizaki M, et al: Enhanced oncolysis by a tropism-modified telomerase-specific replication-selective adenoviral agent OBP-405 ('Telomelysin-RGD'). Oncogene 24: 3130-3140, 2005.

8. Umeoka T, Kawashima T, Kagawa S, et al: Visualization of intrathoracically disseminated solid tumors in mice with optical imaging by telomerase-specific amplification of a transferred green fluorescent protein gene. Cancer Res 64: 6259-6265, 2004.

9. Hashimoto Y, Watanabe Y, Shirakiya Y, et al: Establishment of biological and pharmacokinetic assays of telomerase-specific replication-selective adenovirus. Cancer Sci 99: 385-390, 2008.

10. Bischoff J R, Kirn D H, Williams A, et al: An adenovirus mutant that replicates selectively in p53-deficient human tumor cells. Science 274: 373-376, 1996.

11. Kishimoto H, Kojima T, Watanabe $\mathrm{Y}$, et al: In vivo imaging of lymph node metastasis with telomerase-specific replicationselective adenovirus. Nat Med 12: 1213-1219, 2006.

12. Kurihara Y, Watanabe Y, Onimatsu H, et al: Telomerase-specific virotheranostics for human head and neck cancer. Clin Cancer Res 15: 2335-2343, 2009.
13. He RG, Qiu WL and Zhou XJ: The establishment of Acc-2 and Acc-3 and their morphological observation. J West China Stromatol 6: 1-3, 1986

14. Guan XF, Qiu WL, He RG and Zhou XJ: Selection of adenoid cystic carcinoma cell clone highly metastatic to the lung: an experimental study. Int J Oral Maxillofac Surg 26: 116-119, 1997.

15. Fujiwara T: A novel molecular therapy using bioengineered adenovirus for human gastrointestinal cancer. Acta Med Okayama 65: 151-162, 2011.

16. Yasuda Y,Kondo S, Nagumo T, et al: Anti-tumor activity of dehydroxymethylepoxyquinomicin against human oral squamous cell carcinoma cell lines in vitro and in vivo. Oral Oncol 47: 334-339, 2011.

17. Sakakibara A, Tsukuda M, Kondo N, Tsukamoto H, Mukudai Y, Umezawa K and Shintani S: Examination of the optimal condition on the in vitro sensitivity to telomelysin in head and neck cancer cell lines. Auris Nasus Larynx 38: 589-599, 2011.

18. Kim NW, Piatyszek MA, Prowse KR, et al: Specific association of human telomerase activity with immortal cells and cancer. Science 266: 2011-2015, 1994.

19. Shay JW and Bacchetti S: A survey of telomerase activity in human cancer. Eur J Cancer 33: 787-791, 1997.

20. Dong CK, Masutomi K and Hahn WC: Telomerase: regulation, function and transformation. Crit Rev Oncol Hematol 54: 85-93, 2005.

21. Kyo S, Takakura M, Kohama $\mathrm{T}$ and Inoue $\mathrm{M}$ : Telomerase activity in human endometrium. Cancer Res 57: 610-614, 1997.

22. Kyo $\mathrm{S}$ and Inoue $\mathrm{M}$ : Complex regulatory mechanisms of telomerase activity in normal and cancer cells: how can we apply them for cancer therapy? Oncogene 21: 688-697, 2002.

23. Sasaki T, Tazawa H, Hasei J, et al: Preclinical evaluation of telomerase-specific oncolytic virotherapy for human bone and soft tissue sarcomas. Clin Cancer Res 17: 1828-1838, 2011.

24. Li G, Kawashima H, Ogose A, et al: Efficient virotherapy for osteosarcoma by telomerase-specific oncolytic adenovirus. J Cancer Res Clin Oncol 137: 1037-1051, 2011.

25. Fujiwara T, Kagawa S, Kishimoto H, et al: Enhanced antitumor efficacy of telomerase-selective oncolytic adenoviral agent OBP-401 with docetaxel: preclinical evaluation of chemovirotherapy. Int J Cancer 119: 432-440, 2006.

26. Hioki M, Kagawa S, Fujiwara T, et al: Combination of oncolytic adenovirotherapy and Bax gene therapy in human cancer xenografted models. Potential merits and hurdles for combination therapy. Int J Cancer 122: 2628-2633, 2008.

27. Nakajima O, Matsunaga A, Ichimaru D, Urata Y, Fujiwara T and Kawakami K: Telomerase-specific virotherapy in an animal model of human head and neck cancer. Mol Cancer Ther 8: 171-177, 2009.

28. Yamasaki Y, Tazawa H, Hashimoto Y, et al: A novel apoptotic mechanism of genetically engineered adenovirus-mediated tumour-specific p53 overexpression through E1A-dependent p21 and MDM2 suppression. Eur J Cancer 48: 2282-2291, 2012.

29. Hasei J, Sasaki T, Tazawa H, et al: Dual programmed cell death pathways induced by p53 transactivation overcome resistance to oncolytic adenovirus in human osteosarcoma cells. Mol Cancer Ther 12: 314-325, 2013.

30. Nemunaitis J, Tong AW, Nemunaitis M, et al: A phase I study of telomerase-specific replication competent oncolytic adenovirus (telomelysin) for various solid tumors. Mol Ther 18: 429-434, 2009.

31. Del Vecchio S, Zannetti A, Fonti R, Pace L and Salvatore M: Nuclear imaging in cancer theranostics. Q J Nucl Med Mol Imaging 51: 152-163, 2007. 